\title{
Elimination of Schistosomiasis Japonica in China: From the One Health Perspective
}

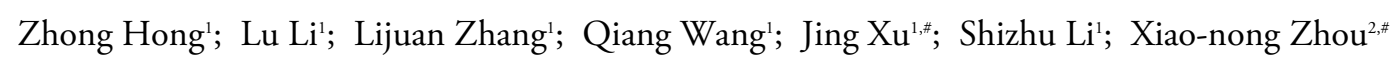

\begin{abstract}
Schistosomiasis japonica is caused by infection of Schistosoma japonicum (S. japonicum), which infected 12 million residents in the 1950s in China and was a heavy burden to public health and socioeconomic development (1). After more than seven decades of effort to control schistosomiasis, the prevalence of schistosomiasis has been reduced dramatically in China. Among the 450 endemic counties (including city and district-level jurisdictions), $74.89 \%$ (337/450), 21.87\% (98/450), and 3.33\% (15/450) have achieved the criteria of elimination, transmission interruption, and transmission control of schistosomiasis, respectively. As the overall endemic status of schistosomiasis remains at a low level, the strategies shifted from snail control to morbidity control and then to an integrated strategy that emphasized infection source control. However, being a vector-borne and zoonotic disease, schistosomiasis japonica is intricately linked to multiple factors including biological, natural, and socioeconomic risk factors. In order to eliminate schistosomiasis earlier and more thoroughly, the One Health approach should be adopted, which focuses on solving complex health problems from a macro-level perspective of interactions among human, animal, and environment, emphasizing multi-institution, interdisciplinary, and cross-regional collaboration and communication.
\end{abstract}

Human schistosomiasis is a water-borne infectious disease caused by infection with blood flukes of the genus Schistosoma. The disease occurs worldwide in 78 countries and regions in Asia, South America, the Middle East, and Africa. Globally, over 780 million people are at risk of infection and 250 million people are infected, of which $90 \%$ are concentrated in subSaharan Africa (2-4). The estimated global burden of schistosomiasis is 3.31 million disability-adjusted life years (DALYs) (5). In China, human schistosomiasis is only caused by $S$. japonicum. Like other humanhosting Schistosoma spp., the life cycle of $S$. japonicum includes adult worm, egg, miracidium, sporocyst, cercariae, and schistosomula, requiring an intermediate host and a definitive host to complete its asexual and sexual production separately (Figure 1). Oncomelania hupensis, an amphibious snail, is the only intermediate host of $S$. japonicum. Humans and over forty other mammals serve as definitive hosts of $S$. japonicum and play a role in spreading infections when they excrete feces containing schistosome eggs to the environment.

The control of schistosomiasis was a high priority in China soon after the founding of the People's Republic of China in 1949, and schistosomiasis japonica was largely brought under control through 7 decades of effort. However, great challenges still exist to completely eliminate schistosomiasis from the country by 2030 due to climate change, natural disasters, socioeconomic development, environmental protection, etc. The progress of the national schistosomiasis control program and the experience accumulated over past several decades in China is reviewed in this article, and solutions to achieve the elimination of schistosomiasis through a One Health approach are explored, which addresses complex health issues from a holistic perspective of human-animalenvironment interaction.

\section{Progress of the National Schistosomiasis Control Program in China}

From Figure 2, we can see that the systematic and large-scale control activities against schistosomiasis have been initiated since the mid-1950s in China. During the whole process, the selection and implementation of control strategies were in accordance with socioeconomic level, the health needs of the people in the epidemic area, and the law of epidemic change. The great achievements in the prevention and control of schistosomiasis in China are 


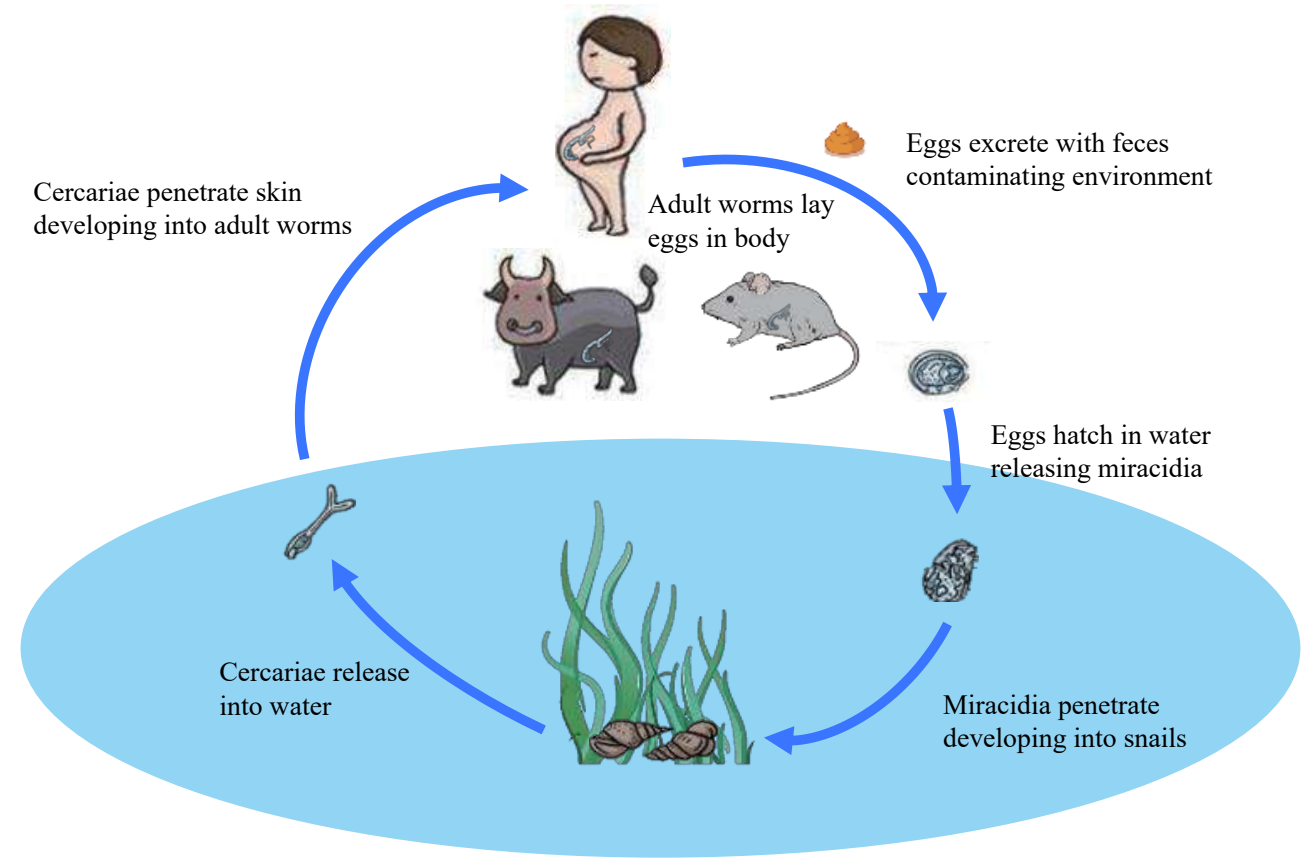

FIGURE 1. The life cycle of S. japonicum.

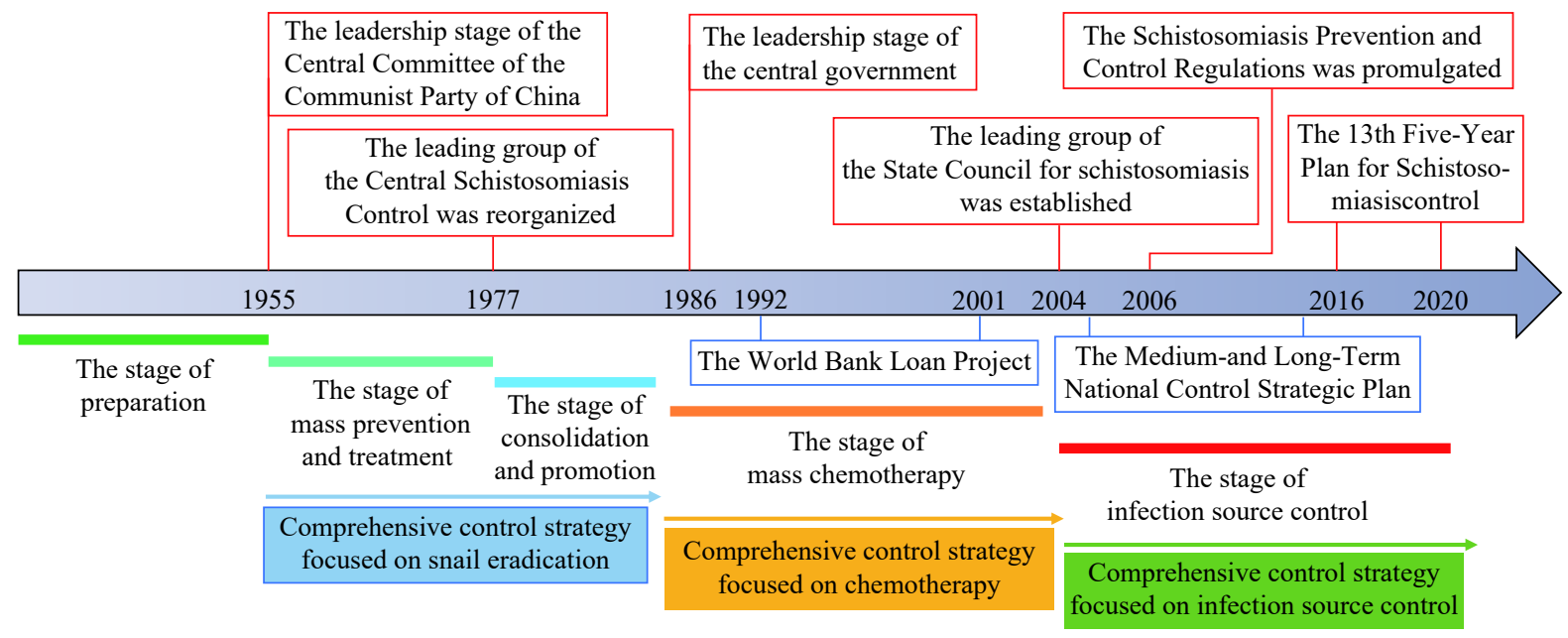

FIGURE 2. Different stages of schistosomiasis control in China.

closely related to the timely adjustment of the control strategy, which has gone through the following three stages.

\section{Strategy Focused on Snail Elimination (mid-1950s to the early 1980s)}

From the 1950 s to the early 1980 s, because of the slow economic growth, scarce health resources, lack of low toxicity medicine, and insufficient understanding of epidemiology towards schistosomiasis, large scale chemotherapy could not be implemented and transmission was difficult to be interrupted. Therefore, snail control was the most suitable way to reduce the damage caused by schistosomiasis. The main countermeasures against schistosomiasis were to eliminate snails through environmental modification in combination with massive agricultural activities and molluscicide using pentachlorophenol sodium and other chemical drugs. In addition, human patients and infected animals were treated, and interventions such as personal protection, feces management, safe water usage, and government publicity were conducted. This strategy had great achievements. The snail breeding areas decreased significantly nationwide, especially in waterway network regions, and 4 provincial-level 
administrative divisions including Shanghai, Guangdong, Guangxi, and Fujian had interrupted the transmission of schistosomiasis by 1985 . However, this strategy required a large quantity of manpower that was not possible after the economic system reforms in the late 1970s. In addition, this strategy was not practical in marshland and lake regions where water levels fluctuated and mountainous areas where economic development was slowed.

\section{Strategy Focused on Morbidity Control} (mid-1980s to 2003)

With the recognition of the impossibility of reaching schistosomiasis elimination under the circumstances at that time and the availability of praziquantel, the drug effective against schistosomiasis that had low toxicity, China adjusted its strategy from snail control to morbidity control following guidelines from the World Health Organization (WHO) and supported by the World Bank Loan Project (WBLP) in the mid-1980s. Mass drug administration (MDA) was conducted simultaneously to human beings and domestic animals, supplemented by snail control and government publicity. The morbidity control strategy was boosted nationwide by the WBLP for schistosomiasis control in China, which spanned from 1992 to 2001 and covered 8 endemic PLADs. The number of cases with schistosome infection decreased from 1.64 million in 1989 to 0.82 million by 2001, with a reduction rate of $49.94 \%$. However, the morbidity control strategy that focused on chemotherapy could not prevent reinfection of schistosomes in human beings and livestock. Moreover, schistosomiasis rebounded significantly after the termination of WBLP, especially after the occurrence of serious flooding in 1998 along the Yangtze River Valley (๑). The lag effect of flood disasters and the implementation of the strategy of "returning farmland to lakes and removing embankments for flood discharge" further increased the distribution area of snails in China, expanded the scope of epidemic areas, and increased the population threatened by infection.

\section{Strategy Focused on Infection Source Control (Since 2004)}

Because of the low-level incidence, a comprehensive strategy was formulated to interrupt transmission via controlling schistosome egg contamination or infectious sources' exposure to the environment, particularly in snail habitats (7). In addition to implementing conventional countermeasures such as chemotherapy on human beings and livestock, molluscicide, and health education, interventions focusing on the control of the source of infection were strengthened, such as raising livestock in pens, herding bovine in snail-free areas (avoiding eggs polluting the snail environment), replacing bovine with machines (reducing the number and kind of susceptible animals), reconstructing the sanitary toilets (conducting harmless treatment of excrement), and collecting the excrement of fishermen (avoiding eggs polluting water). In addition, engineering projects conducted by local ministries of agriculture, forestry, water conservancy, land resources, and others were integrated with the snail control projects to remove the snail breeding areas and prevent environmental pollution from the feces of human beings and animals (8). China reached transmission control criteria issued by the Chinese government as well as criteria of schistosomiasis elimination as a public health problem defined by the WHO by 2015.

\section{Perspectives to Eliminate Schistosomiasis Through the One Health Approach}

Being a vector-borne and zoonotic disease, schistosomiasis japonica is intricately linked to multiple factors including biological, natural, and socioeconomic risk factors, such as widespread snail habitats, a variety of animal hosts, frequent occurrences of floods, increased population mobilization, and transportation of goods, which are all threatening the obtained achievements and hindering the process of schistosomiasis elimination. The One Health approach, focusing on solving complex health problems from the overall perspective of humananimal-environmental interaction, advocates for multisectoral, cross-regional, and trans-disciplinary collaborations in all aspects $(9-10)$. Thus, to realize the goal set by the strategic plan of healthy China 2030 to eliminate schistosomiasis in China, the One Health approach should be applied in national control program.

\section{Integrated Surveillance and Response System}

It was reported that about $75 \%$ of emerging infectious diseases of humans have emerged from animals during the past few decades (11). Thus, the 
importance of surveillance of schistosome-susceptible animals should not be neglected. Therefore, there must be a merger of monitoring clinical practice, veterinary practice, vector surveillance, and environmental surveillance to more effectively evaluate and plan an appropriate response to the transmission of schistosomiasis. In order to promote integrated surveillance and response systems, general reference laboratories, surveillance centers based on epidemiological and big data related to schistosomiasis would be useful for pooling resources and providing rapid access information (5). Some surveillance indicators, such as observed risk factors, newly identified infected cases in humans and animals, and infection status of wild animals following examination, would allow for rapid responses before an outbreak occurs (12).

\section{Multisectoral Collaboration}

The transmission and distribution of schistosomiasis are influenced by many types of factors including the environments, pathogens, vectors, local economic conditions, and level of societal development. Interventions such as blocking the transmission of schistosomiasis from infection source to surroundings, treating or culling infected livestock, raising schistosome-unsusceptible poultries, replacing cattle with machines, and herding livestock in snail-free areas are imperative in order to control animal reservoir hosts. All of these need veterinarians, livestock producers, health workers, and political decisionmakers to work together. For snail control, there are two major approaches, which are chemical control by molluscicide and environmental modification. The former faces great challenges due to increasing environmental protection laws that prohibit controlling snail habitats along the Yangtze River and wetland eco-zones. The latter mainly relies on the projects being chaired by the ministries of water conservancy, agriculture, forestry, land resources, etc. (13).

\section{Interdisciplinary Research}

Interdisciplinary research, with the collaboration of scientists in the field of biology, ecology, computer science, and more, is a key component in understanding the complex transmission patterns and exploring interventions against schistosomiasis. Understanding the transmission dynamics of schistosomiasis in human-environment-animal interface will benefit schistosomiasis elimination (14).
Furthermore, interdisciplinary research will help connect schistosomiasis control with information technology and big data organically, establishing an efficient and sensitive early warning system worldwide (15). Moreover, increased emphasis on comparative diseases etiology, pathology, genomics, proteomics, and metabolomics is important to potentially discover novel treatments for humans and animals.

\section{CONCLUSION}

Schistosomiasis japonica is a vector-borne zoonotic disease, requiring integrated approaches for prevention and elimination to deal with the relationship among animals, the environment, and humans. Although great achievements have been reached in China through more than 70 years of effort, the elimination of schistosomiasis is a constant challenge complicated by many different biological and environmental factors involved in the circulation of the diseases, including widespread snail habitats, various animal hosts, frequent occurrence of flooding, and increased population mobilization and goods transportation. As human influence on the environment increases and the transmission of disease becomes more complex, adopting the One Health approach is an essential key to elimination of this disease. Therefore, strengthening multi-institution, interdisciplinary, and cross-regional cooperation and conducting further research to this disease are important to accelerate the process of schistosomiasis elimination.

Conflicts of interest: No conflicts of interest.

Funding: Supported by the National Science Foundation of China (Grant No. 82073619), the Fifth Round of Three-Year Public Health Action Plan of Shanghai (No. GWV-10.1-XK13).

doi: $10.46234 / \mathrm{ccdcw} 2022.024$

\# Corresponding authors: Jing Xu, xujing@nipd.chinacdc.cn; Xiaonong Zhou, zhouxn1@chinacdc.cn.

${ }^{1}$ National Institute of Parasitic Diseases, Chinese Center for Disease Control and Prevention (Chinese Center for Tropical Diseases Research); NHC Key Laboratory of Parasite and Vector Biology; WHO Collaborating Centre for Tropical Diseases; National Center for International Research on Tropical Diseases, Shanghai, China; ${ }^{2}$ School of Global Health, Chinese Center for Tropical Diseases Research, Shanghai Jiao Tong University School of Medicine, Shanghai, China.

Submitted: December 03, 2021; Accepted: January 11, 2022

\section{REFERENCES}

1. Li YL, Dang H, Zhang LJ, Li GP, Shan XW, Yuan M, et al. 
Schistosomiasis surveillance - China, 2015-2018. China CDC Wkly 2020;2(3):39 - 43. http://dx.doi.org/10.46234/ccdcw2020.011.

2. Colley DG, Bustinduy AL, Secor WE, King CH. Human schistosomiasis. Lancet 2014;383(9936):2253 - 64. http://dx.doi.org/ 10.1016/S0140-6736(13)61949-2.

3. Deol AK, Fleming FM, Calvo-Urbano B, Walker M, Bucumi V, Gnandou I, et al. Schistosomiasis-assessing progress toward the 2020 and 2025 global goals. N Engl J Med 2019;381(26):2519 - 28. http:// dx.doi.org/10.1056/NEJMoa1812165.

4. Ekpo UF, Oluwole AS, Abe EM, Etta HE, Olamiju F, Mafiana CF. Schistosomiasis in infants and pre-school-aged children in sub-Saharan Africa: implication for control. Parasitology 2012;139(7):835 - 41. http://dx.doi.org/10.1017/S0031182012000029.

5. Pisarski K. The global burden of disease of zoonotic parasitic diseases: top 5 contenders for priority consideration. Trop Med Infect Dis 2019;4(1):44. http://dx.doi.org/10.3390/tropicalmed4010044.

6. Wang RB, Wang TP, Wang LY, Guo JG, Yu Q, Xu J, et al. Study on the re-emerging situation of schistosomiasis epidemics in areas already under control and interruption. Chin J Epidemiol 2004;25(7):564 - 7. http://dx.doi.org/10.3760/j.issn:0254-6450.2004.07.004. (In Chinese).

7. Wang LD, Chen HG, Guo JG, Zeng XJ, Hong XL, Xiong JJ, et al. A strategy to control transmission of Schistosoma japonicum in China. N Engl J Med 2009;360(2):121 - 8. http://dx.doi.org/10.1056/ NEJMoa0800135.

8. Wang TP, Cao ZG, Chen HG, Zhou XN. Changes of control strategy and improvement of schistosomiasis control in China. Chin J Schisto Control 2009;21(4):241 - 2. http://dx.doi.org/10.3969/j.issn.10056661.2009.04.001. (In Chinese).
9. Wang TP. Thinking on the control strategy at schistosomiasis elimination stage in China. Chin J Schisto Control 2019;31(4):358 61. http://dx.doi.org/10.16250/j.32.1374.2019162. (In Chinese).

10. Hong A, Zampieri RA, Shaw JJ, Floeter-Winter LM, Laranjeira-Silva MF. One health approach to leishmaniases: understanding the disease dynamics through diagnostic tools. Pathogens 2020;9(10):809. http:// dx.doi.org/10.3390/pathogens 9100809 .

11. Elelu N, Aiyedun JO, Mohammed IG, Oludairo OO, Odetokun IA, Mohammed KM, et al. Neglected zoonotic diseases in Nigeria: role of the public health veterinarian. Pan Afr Med J 2019;32:36. http://dx. doi.org/10.11604/pamj.2019.32.36.15659.

12. Wang W, Bergquist R, King $\mathrm{CH}$, Yang K. Elimination of schistosomiasis in China: current status and future prospects. PLoS Negl Trop Dis 2021;15(8):e0009578. http://dx.doi.org/10.1371/ journal.pntd.0009578.

13. Yang F, Xu J, Lü S, Cao CL, Li SZ, Zhang LJ. Analysis on epidemiological characteristics of current advanced schistosomiasis cases in China based on the Epidemiological Dynamic Data Collection Platform (EDDC). Chin J Schisto Control 2021;33(3):234 - 9,247. http://dx.doi.org/10.16250/j.32.1374.2021113. (In Chinese).

14. Wang W, Yang K. Implementation of precision control to facilitate the progress towards schistosomiasis elimination in China. China Trop Med 2020;20(7):595 - 8. http://dx.doi.org/10.13604/j.cnki.46-1064/r. 2020.07.01. (In Chinese).

15. Hong Z, Wu LL, Wang LP, Xu J. Progress and challenges on global schistosomiasis control. Chin J Parasitol Parasit Dis 2021;39(3):514 9. http://dx.doi.org/10.12140/j.issn.1000-7423.2021.04.015. (In Chinese). 